\title{
REVISIÓN DE LA INVERSIÓN SUSTENTABLE EN LA BOLSA MEXICANA DURANTE PERIODOS DE CRISIS.
}

\author{
Oscar V. De la Torre Torres* \\ Universidad Michoacana de San Nicolás de Hidalgo, Facultad de Contaduría y Ciencias Administrativas \\ Ma. Isabel Martínez Torre-Enciso \\ Universidad Autónoma de Madrid, Departamento de Financiación e Investigación Comercial \\ (Recibido 07 de octubre 2014, aceptado 28 de marzo 2015)
}

\begin{abstract}
Resumen
El presente trabajo estudia la eficiencia media-varianza de la inversión sustentable (IS) en México. Esto al comparar el índice IPC sustentable (IPCS) con el IPC compuesto (IPCcomp). Utilizando valores de periodicidad diarios de noviembre de 2008 a septiembre de 2014, así como un modelo CAPM estándar, la prueba de expansión de Huberman y Kandel (1987) y un modelo $\mathrm{AR}(0)$ markoviano de cambio de régimen, el presente estudio demuestra que existe una igualdad estadística en el desempeño del IPCS y el IPCcomp. Esto sugiere que la IS (como estilo de inversión) es un buen sustituto ante la inversión convencional en un índice de mercado más amplio. Esto último sin exponer al inversionista a una pérdida significativa de eficiencia media-varianza. Las dos aportaciones torales del trabajo son 1) que es de los primeros en realizarse en la inversión sustentable mexicana y 2) que los resultados se sostienen ante la presencia de dos regímenes de volatilidad.
\end{abstract}

\section{Abstract}

The present paper studies the mean-variance efficiency of sustainable investment (SI) in Mexico by testing the IPC sustainability (IPCS) index against the broad market IPCcomp one. Using daily index values from November 2008 to September 2014, along with a standard CAPM Model, a Huberman and Kandel (1987) CAPM spanning test and an AR(0) Markov-Switching Model, the present study shows a statistically equal performance between the IPCS and the IPCcomp, suggesting SI (as investment style) as a good substitute of the broad market one without a significant loss of mean-variance efficiency. The two breakthroughs of the present paper are 1) these tests are the first ones of its type in the Mexican Sustainable investment and 2) the fact that our conclusions give support to SI in the presence of two volatility regimes.

Clasificación JEL: C580, G11, G170, G23

Palabras clave: Modelos Markovianos de Cambio de Régimen, Diversificación, Selección de Portafolios, Simulación y Pronostico Financiero, Inversión ética, Sustentabilidad.

* Facultad de Contaduría y Ciencias Administrativas, Universidad Michoacana de San Nicolás de Hidalgo Ave. Francisco J. Mújica S/N Edificio AII Col. Felicitas del Río Morelia Michoacán C.P. 58030. Correo electrónico:oscar.delatorre.torres@gmail.com 


\section{Introducción}

La inversión sustentable (SI) en bolsa es una actividad que en las últimas décadas ha tomado notable interés. Esto debido a una mayor preocupación de la sociedad en general (en especial a nivel internacional) por fomentar las sanas prácticas de trabajo en las empresas y en la economía, dando validez a conceptos teórico-económicos propios de la Teoría de Juegos como son el equilibrio entre los intereses de los individuos de una sociedad o la constructos de la Teoría de las partes interesadas (stakeholder theory) de Freeman (1984). Esto, a su vez, lleva a conceptos como la responsabilidad social o sustentabilidad (cuyo significado es sinónimo para los fines del presente).

Las primeras prácticas de inversión socialmente responsable se pueden encontrar en escritos religiosos como son la Torá, el Corán o la propia biblia. De ellos emanan prácticas sociales y religiosas en la actividad empresarial y la inversión como la Ley Sharia que orienta el proceder personal y, por ende, financiero de los adeptos a esta religión. Otro ejemplo notorio se aprecia con las prácticas que durante los siglos XVII a XIX llevaron a cabo grupos cristianos como los puritanos, los cuáqueros o los metodistas, quienes no invertían en empresas relacionadas al alcohol, la venta de armas o el tráfico de esclavos. En una historia más contemporánea, la inversión sustentable fue adoptada en los mercados financieros en la década de 1970 (con fondos como el PAX fund en 1971). Esto como un movimiento activista contra la guerra de Vietnam, siendo afectadas empresas como Dow Chemical quien fuera criticada fuertemente por la invención del Napalm. En los años 80's, el activismo se tornó en contra de la inversión hacia empresas que tenían filiales o base en Sudáfrica y de las cuales se tenía sospecha que estaban involucradas en el apartheid. Por último, durante la misma década, la inversión sustentable tomó la forma que se conoce hoy en día cuando múltiples fondos de pensiones como el California Public Employees Retirement System (CalPERS) enfocaron su atención a invertir en empresas que no se relacionaran con malas prácticas de gobierno corporativo, malas relaciones con empleados y proveedores o empresas relacionadas con giros de alto impacto ambiental.

Derivado de esta tendencia, organismos internacionales como la Organización de las Naciones Unidas, la OCDE y el Banco Mundial han adoptado, junto con firmas consultoras como MSCI (antes KLD) o Eiris, criterios de evaluación de la responsabilidad social (o sustentabilidad) en las empresas. Esto basándose en los tres pilares propuestos por las Naciones Unidas de Gobierno corporativo, Impacto ambiental e impacto social (proveedores, trabajadores, etc.). Con la aparición de estas iniciativas y con las prácticas de inversión observadas en los años 80 , se desarrollaron métodos de selección de portafolios sustentables a través de métodos de selección positiva (positive social screennig) que consisten en incluir acciones de empresas que cubren niveles altos de sustentabilidad o métodos de selección negativa (negative social screening), que se basan en la omisión de acciones "pecaminosas". Esto último en el contexto de la selección de portafolios. De estas prácticas, múltiples índices prestigiosos como el Dow Jones Sustainability index, el Domini 4000 o el FTSE4good aparecieron como benchmarks de desempeño de las acciones que cumplen con determinados criterios de sustentabilidad. Derivado de esto y como 
se verá a continuación en la revisión de bibliografía previa, múltiples estudios se han realizado sobre la inversión sustentable.

De manera consecuente con lo anterior y para el caso mexicano, se observa que la práctica de la IS es relativamente joven en el país. Sin embargo, esta tendencia ha sido materia de interés, al grado de que en el año 2006 el Centro de Excelencia en Gobierno Corporativo de la Universidad Anahuac del Sur y la consultora EIRIS unieron sus esfuerzos con la Bolsa Mexicana de Valores para calificar la sustentabilidad de empresas que cotizan en México. Dado este esfuerzo conjunto, en el año 2010 se lanza al mercado el primer índice sustentable: el IPC sustentable o IPCS del cual se tiene información histórica desde el 28 de noviembre del 2008.

Ante la relevancia de este estilo de inversión (el sustentable) y ante críticas teóricas como las primigenias de Langbein y Posner (1980), se tiene como principal interés estudiar la eficiencia media-varianza de la inversión sustentable en México. Esto por que se parte del hecho de que, si se siguen los postulados de la Teoría Moderna de Portafolios, una cartera formada con un subconjunto no podrá ser más eficiente (en los términos citados) que una proveniente del conjunto mayor. Esto como es el caso de un portafolio de todo un mercado. Esto es: es de esperarse que la relación riesgo-rendimiento sea menor en un índice sustentable que en un subconjunto más amplio como es un índice de mercado representado, para el presente trabajo, por el IPC compuesto (IPCcomp).

Una vez planteado el objetivo principal de los trabajos realizados, se observa que el presente artículo iniciara con una revisión de los estudios y bibliografía relativos a la inversión sustentable. Esto seguido de la exposición de la metodología y resultados que comprobarán la hipótesis de trabajo (a mencionar en breve). Una vez hecho esto, el documento termina con las conclusiones y las guías para investigación futura.

\section{Revisión de la literatura}

La inversión sustentable (IS) en bolsa ha sido estudiada en múltiples países. El primer estudio académico lo realiza Moskowitz (1972) quien revisa el desempeño (rendimiento) de los fondos de IS. Sus resultados observan que este tipo de fondos tienen un desempeño superior en comparación a fondos cuya política de inversión es replicar un índice de mercado como es el S\&P500. Esto último lo atribuye a que el mercado no tenía, en aquello entonces, valuada la responsabilidad social en las acciones de una empresa.

Por otro lado, Statman (2000) realiza una de las investigaciones más citadas al contrastar, empleando el estadístico de Modigliani y Modigliani (1997), el desempeño del índice de IS Domini 4000 con el índice S\&P500. De manera complementaria, estudió el desempeño de fondos de IS. Sus resultados prueban que a pesar de que el desempeño de los fondos de IS tienen un mejor desempeño que los no sustentables, no existen evidencias estadísticas para verificar este resultado. De manera complementaria, Boutin-Doufresne y Savaria (2004) estudiaron el desempeño de fondos canadienses de IS, comparándolos con fondos de inversión e índices de mercado (como el S\&PTSX). Las conclusiones a las que llegaron fueron las mismas que las presentadas en el estudio de Statman, resaltando que este tipo de fondos tienen un menor riesgo diversificable. 


\section{Nueva Época REMEF (The Mexican Journal of Economics and Finance)}

De manera similar, Schröder (2004) analizó el desempeño de 56 fondos de IS de Estados Unidos, Alemania y Suiza, así como 10 índices de IS. Al emplear la alpha de Jensen con datos del año 2000 a 2002, demostró que tampoco existe evidencia estadística de un desempeño superior contra la inversión convencional.

Partiendo de lo estudiado por Schröder, Bauer, Koedijk y Otten (2005) revisaron el desempeño de fondos de IS alemanes, estadounidenses e ingleses, comparándolos con sus respectivos índices de mercado y empleando el modelo multifactor de Carhart (1997), el cual es una extensión del reconocido modelo de Fama y French (1992). Los resultados de esta prueba demostraron que no existe un desempeño superior de la IS respecto a la inversión común (de mercado). De manera complementaria, observaron que los fondos estadounidenses tienen una gran concentración en acciones de alta cotización y prestigio (blue chips); mientras que los fondos ingleses y alemanes tienen una preferencia por las acciones de menor capitalización. De manera análoga a Bauer, Koedjik y Otten, Scholtens (2005) estudió el desempeño de fondos holandeses contra el índice AEX, empleando el modelo de Carhart. Como el caso anterior, tampoco encontró un desempeño superior estadísticamente significativo.

Hasta el momento, la revisión de literatura se ha enfocado a ver la significancia estadística de la alfa de un modelo estándar o multifactorial de valuación de activos. Dado esto, se tiene como principal punto de partida e interés en el presente trabajo el replicar las pruebas anteriores, siendo el primer paso el demostrar la significancia estadística de la alfa o desempeño superior del IPCS respecto al mercado (IPCcomp). Esto es así por que en México no existen índices de crecimiento o valor y por que las metodologías de los índices de mercado por tamaño de capitalización están en un proceso de revisión (junto con el desarrollo de otros índices) por parte de los autores del presente trabajo. Ante esta limitante, el desarrollo de modelos como el de Fama y French o el de Carhart, quedarán como interés de trabajos futuros y complementarios al presente.

Para complementar la revisión anterior, así como para dar mayor solidez a las pruebas por realizar, se observa que Schröder (2007) realizó pruebas a 29 índices mundiales de sustentabilidad al emplear la prueba (HK) de expansión de Huberman y Kandel (1987). Con esta buscó demostrar la siguiente hipótesis nula $\alpha=0, \beta=1$. Éste autor también empleó una versión propia del modelo de Fama y French, al resolver el sistema de ecuaciones de las regresiones estudiadas con el método de regresiones aparentemente no relacionadas (seemingly unrelated regression). Con sus pruebas, Schröder no encontró, al igual que en su estudio del año 2004, evidencia de un desempeño superior de los índices de IS contra los índices de mercado. De manera complementaria, con la prueba de expansión de HK, encontró que, al no sostenerse la hipótesis nula, el índice de mercado no puede replicar, de manera sintética el índice sustentable y por tanto, ambos índices no pueden ser sustitutos mutuos en un proceso de selección de portafolios. Es decir, no pueden utilizarse de manera indistinta.

Ante la simplicidad de la prueba de hipótesis, así como en un deseo de demostrar que la inversión sustentable no lleva a una eficiencia media-varianza inferior en la inversión sustentable respecto a la inversión de mercado, se observa 
el interés de los autores de incorporar la prueba de expansión de Huberman y Kandel (HK). Esto en un afán de demostrar que el IPCS puede ser un buen sustituto de la inversión de mercado (IPCcomp) sin pérdida de eficiencia mediavarianza (alfa negativa).

De manera complementaria a los estudios previos, Capelle-Blancard y Couderc (2009) realizaron un estudio de evento (event study) al observar el efecto en el desempeño de las acciones que eran incluidas o excluidas de índices sustentables. Los resultados de su trabajo encontraron que el impacto en la valuación de las acciones solo se recibía en el corto plazo de manera inmediata al anuncio pero, que en el largo plazo, la influencia de este efecto no era observable.

Con una perspectiva global, Lee y Faff (2009) contrastaron el índice DJ global contra el DJ de sustentable. Esto al reproducir índices con selección de acciones sustentables con un nivel alto en su medida de sustentabilidad (leading social screening), así como acciones con niveles bajos (lagging social screenning). Estos índices los compararon con índices formados con acciones que no eran sustentables pero del mismo giro o perfil, así como con el índice de mercado mismo (DJ Global). Para realizar sus pruebas, emplearon un modelo de valuación de activos de seis factores que utilizó, de entre ellos, la influencia del mercado global, el valor en libros, el valor de capitalización, el momentum y el efecto país y de sector industrial. Sus resultados llevaron a no encontrar una alfa significativa, complementando estudios previos que sugieren que el mercado no incorpora la sustentabilidad en la valuación de las acciones de una compañía.

En un deseo de dar los primeros pasos conductistas, Jansson et.al. (2011) estudiaron la IS al determinar los motivadores de la adopción de la misma en Suecia. Ellos encuestaron a los tres bancos más grandes de ese país, así como a cuatro fondos de pensiones y a prácticamente todos los fondos de inversión enfocados a IS. Al emplear el método de componentes principales y el análisis discriminante, ellos formaron un factor denominado "regulación" y otro llamado "comportamiento de rebaño" (herd behavior). Su principal conclusión observa que los inversionistas adoptan la sustentabilidad siguiendo un comportamiento de rebaño y que, para los casos de fondos o instituciones que no siguen políticas de sustentabilidad, la regulación es un factor que influye fuertemente para optar por este estilo de inversión.

Por último, empleando modelos markovianos de cambio de régimen (Markov Switching Models) Areal et.al. (2013) emplean el modelo de Carhart y un modelo CAPM condicional en datos de fondos de IS para valuar su desempeño en contraste del S\&P500 ante dos regímenes de volatilidad (alta y baja). Esto con datos de octubre de 1993 a septiembre de 2009. Esto lo hacen al formar tres portafolios con nivel homogéneo de inversión $(1 / n)$ en acciones no sustentables o acciones de empresas con criterios de selección orden religioso y de orden social. Sus resultados llevan a la conclusión de que si bien la inversión sustentable no lleva a rendimientos superiores e la convencional, en periodos de alta volatilidad, el nivel de desempeño y de riesgo en la inversión no sustentable es inferior al caso que si lo es.

De esta revisión no exhaustiva de bibliografía, se desea destacar los casos de Schröder (2007) y Areal et.al (2013). Esto es así porque, partiendo de la crítica teórica de Langbein y Postner (1980) se tendría el objetivo de verificar 
tres argumentos que le darían utilidad práctica a la inversión sustentable en México:

1.- Si bien no se gana eficiencia media-varianza (performance o desempeño) con la inversión sustentable respecto a la inversión convencional o indizada al desempeño de un mercado, es de necesidad demostrar que tampoco se tienen desempeños desfavorables o negativos.

2.- Por otro lado, como una medida para reforzar el empleo de la inversión sustentable, se observa que es más económico (en términos de costos financieros y operatividad) replicar un portafolio o índice sustentable, el cual tiene una cardinalidad menor a la de un índice de mercado. Dado esto, es de interés saber si el último (IPCcomp) tiene un desempeño igual $(\beta=1)$ al IPCS. Si esto es así, entonces se llegará a observar que el empleo del IPCcomp y el IPCS es indistinto, dando fundamento al hecho de que la inversión sustentable en México es un buen sustituto de la inversión indizada a un mercado (IPCcomp).

3.- Por último se buscara demostrar que el rendimiento medio y el nivel de riesgo son favorables para la inversión sustentable en tiempos de alta y baja volatilidad en los mercados y que incluso las probabilidades de tener periodos de alta volatilidad son mayores en la inversión convencional (IPCcomp) que en la sustentable.

Ante el deseo de verificar estos tres argumentos y partiendo de los estudios citados en la presente revisión, se observa que se empleará la prueba de significancia de la alfa de un modelo CAPM estándar, la prueba de expansión de Huberman y Kandel (1987) y modelos markovianos de cambio de régimen para contrastar el comportamiento del IPCS y el IPCcomp en dos regímenes de volatilidad. Lo anterior llevará a los autores a establecer la siguiente hipótesis:

"Emplear el IPCS es igual de benéfico, en términos de eficiencia mediavarianza, que el replicar un índice de mercado como el IPCcomp".

Una vez establecida la revisión de bibliografía y la hipótesis de trabajo, se procederá a la descripción de la metodología empleada, así como de los resultados observados en las pruebas realizadas.

\section{Metodología y resultados observados}

Para demostrar la hipótesis de trabajo se utilizaron los valores históricos diarios del IPCS y el IPCcomp con datos proporcionados por la Bolsa Mexicana de Valores a través de Economática. La ventana temporal empleada fue del 28 de noviembre de 2008 al 20 de septiembre de 2014. Estos datos fueron tratados calculando la variación porcentual $(\Delta \% I)$ de ambos índices $\left(I_{(i, t)}\right)$ a través de la siguiente expresión:

$$
\Delta \% I_{i, t}=\frac{\left(I_{i, t}-I_{i, t-1}\right)}{I_{i, t-1}}
$$

Una vez realizado esto, se programó una rutina informática en MATLAB y se calculó, de manera diaria, la desviación estándar de (1) para los últimos 30 días. Esto para verificar si los niveles de riesgo del IPCS son mayores o no a los del IPCcomp. Complementario a esto, se utilizaron los valores de (1) para realizar múltiples pruebas. Para la primera de ellas se aplicó una prueba 
de significancia de parámetros a un modelo CAPM estándar a través de la siguiente expresión:

$$
\Delta \% I P C S_{t}=\alpha+\beta \Delta \% I P C \operatorname{comp} p_{t}+\varepsilon
$$

En base a (2), es de interés de los autores resaltar si existe un desempeño o performance en la inversión sustentable que sea igual o mayor a la inversión convencional. Esto es, demostrar que $\alpha=0$. De verificarse esto se tienen argumentos a favor de la inversión sustentable (IPCS) en México ya que si bien no se genera un desempeño estadísticamente superior, tampoco se tiene la limitante de generar un desempeño inferior. Como una nota metodológica, se observa que, para lidiar con los problemas de auto-correlación y heteroscedasticidad, se estimó la matriz de covarianzas de los parámetros en (2) con el método de estimadores robustos de Newey y West (1987).

Siguiendo lo realizado por Schröder (2007) y para complementar el resultado anterior, los autores aplicaron la prueba de expansión de Huberman y Kandel (1987). Esto con la finalidad de demostrar que el comportamiento del IPCS y el IPCcomp (es decir la inversión sustentable y la convencional) son estadísticamente iguales. Esto lleva a demostrar la siguiente hipótesis nula a los parámetros de (2): $H_{0}: \alpha=0, \beta=1$. De verificarse la misma, se pueden tener elementos cuantitativos suficientes para afirmar que el empleo de la sustentable y la convencional es el mismo y, por tanto, se puede preferir la primera respecto a la segunda. Esto para fines de contribuir a la práctica de la responsabilidad social empresarial sin atentar a los resultados de los inversionistas. Es decir, se tendrá una conciliación entre la teoría de los accionistas (stock-holders theory) y la de las partes interesadas (stake-holder theory).

Por último, para verificar lo robusto de los resultados así como el desempeño de la inversión sustentable, se empleó un modelo markoviano de cambios de régimen, a través de un filtro de Hamilton (1989) dado por la siguiente expresión:

$$
\Delta \% I_{t}=\mu_{1}+\mu_{2} z_{t}+\left(\sigma_{1}^{2}+\phi z_{t}\right)^{1 / 2} u_{t}
$$

En la misma se tiene una media $\left(\mu_{1}+\mu_{2}\right)$ o valor esperado así como una varianza $\left(\sigma_{1}^{2}+\phi z_{t}\right)$ de la variación porcentual de los índices estudiados que puede darse en un régimen volatilidad baja $\left(z_{t}=0\right)$ o un escenario de alta $\left(z_{t}=1\right)$. Partiendo de que se trata de un filtro de Hamilton, se tendría que sigue un proceso o cadena markoviana de primer orden:

$$
\begin{gathered}
\operatorname{prob}\left[z_{t}=1 \mid z_{t-1}=1\right]=\rho_{11} \\
\operatorname{prob}\left[z_{t}=0 \mid z_{t-1}=1\right]=1-\rho_{11}=\rho_{12} \\
\operatorname{prob}\left[z_{t}=0 \mid z_{t-1}=0\right]=\rho_{22} \\
\operatorname{prob}\left[z_{t}=1 \mid z_{t-1}=0\right]=1-\rho_{22}=\rho_{21}
\end{gathered}
$$


Esto se puede expresar en una matriz de probabilidades de transición la cual también se evaluará con el modelo:

$$
\mathbf{P}=\left[\begin{array}{cc}
\rho_{11} & \rho_{12} \\
\rho_{21} & \rho_{2}
\end{array}\right]
$$

Para poder realizar el modelo dado en (3) se empleó la rutina informática desarrollada por Perlin (2012) para MATLAB.

El objetivo real de utilizar el filtro de Hamilton dado en (3) es separar las observaciones de $\Delta \% I_{t}$ en periodos de crisis o de alta volatilidad de los casos en los que no se da. Dado esto, se busca determinar primero que el rendimiento esperado en periodos de alta volatilidad es mayor para el IPCS que para el IPCcomp y por otro lado observar que, a pesar de que el IPCS pueda tener niveles de desviación estándar mayores en ambos regímenes, el rendimiento es mayor.

\section{Discusión de resultados}

En la figura 1 se expone el comportamiento histórico de los dos índices estudiados con una base 100 al 28 de noviembre de 2008. Estos son comparados contra el desempeño de un portafolio que invirtió en reportos libre de impuesto y costo financiero en CETES de 28 días. Como se puede apreciar, el desempeño del IPCS como benchmark de la inversión sustentable es superior a la inversión común o de mercado (IPCcomp).

Gráfica 1. Desempeño de los dos índices estudiados.

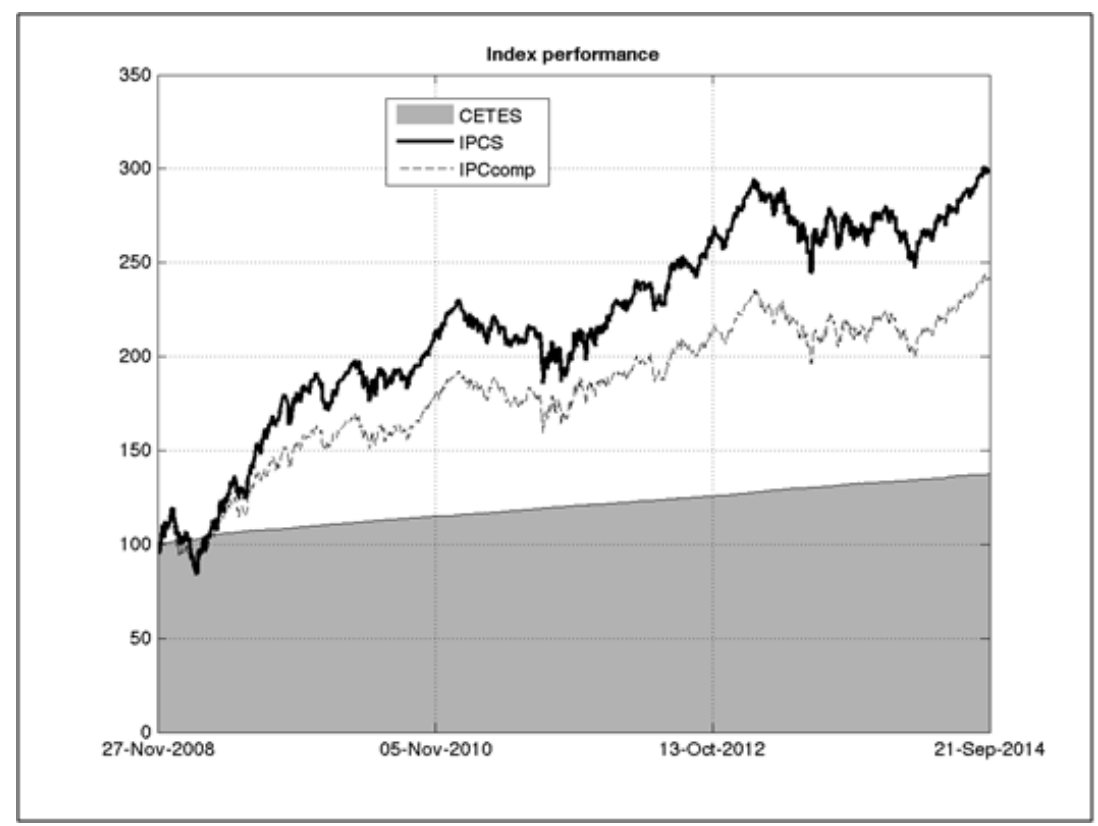

Fuente: Elaboración propia en base a información de la Bolsa Mexicana de Valores y Economática. 
En la gráfica 2 se presentan las variaciones porcentuales calculadas con (1) para los dos índices revisados. Se puede apreciar en la misma que la inversión convencional (IPCcomp) tiene mayores fluctuaciones a lo largo del periodo estudiado. Sin embargo, a pesar de esto, en la gráfica 3 se presenta el histórico de las desviaciones estándar de ambos índices y se puede apreciar que, a pesar de que el IPCS, (consistente con lo teóricamente esperado) tiene niveles de riesgo superiores. A pesar de lo antererior, la ilustración sugiere que estos pueden no ser estadísticamente diferentes.

Gráfica 2. Desempeño de la variación porcentual de los dos índices estudiados.

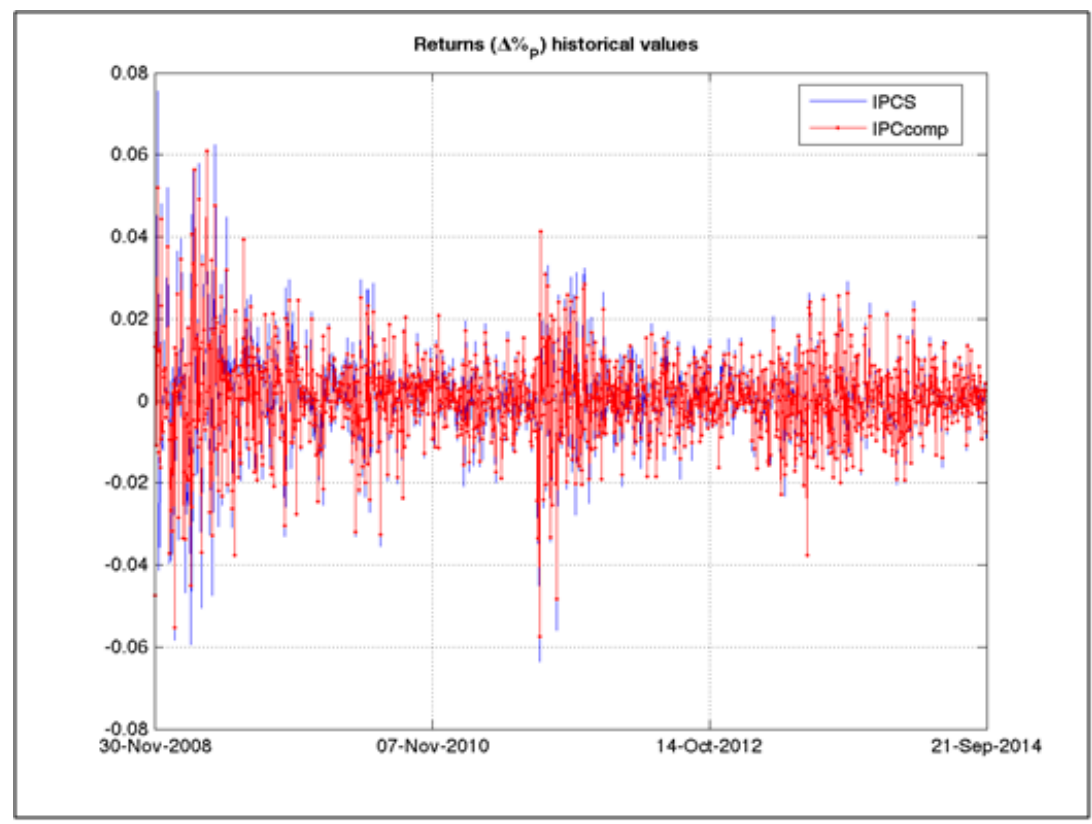

Fuente: Elaboración propia en base a información de las corridas y simulaciones realizadas.

Tabla 1. Prueba de significancia del modelo CAPM estándar con la variación porcentual del IPCS como regresada.

\begin{tabular}{|l|l|l|l|}
\hline Coeficiente & Valor & Estadistico $t$ & Probabilidad \\
\hline$\alpha$ & 0.0001 & 1.1887 & $19.6762 \%$ \\
\hline$\beta$ & 1.0951 & 74.5596 & $0.0000 \%$ \\
\hline Estat.F & $22,329.3284$ & F prob. & $0.0000 \%$ \\
\hline $\mathrm{R}^{2}$ ajust. & 0.9387 & Error std.resid. & $0.3072 \%$ \\
\hline
\end{tabular}

Fuente: Elaboración propia en base a información de las corridas y simulaciones realizadas. 


\section{Nueva Época REMEF (The Mexican Journal of Economics and Finance)}

Hasta el momento se aprecia que el IPCS presenta mayores niveles de riesgo que el IPCcomp en el periodo estudiado pero esto se compensa significativamente con un rendimiento superior. Para corroborar si esto se mantiene en el largo plazo y si este rendimiento superior es significativo, se corrió el modelo CAPM estándar dado en (2) y se llegó a los resultados presentados en la tabla 1.

Como se puede apreciar, el valor de $\alpha$ no es significativo, lo que sugiere que si bien la gráfica 1 lleva a un desempeño superior, este podría ser un fenómeno de corto plazo. Esta situación está en línea con la mayoría de los estudios presentados en la revisión de bibliografía. Sin embargo, es de necesidad señalar que el resultado de la tabla 1 aplica para todo el periodo de estudio y no distingue periodos de alta y baja volatilidad, lo que refuerza el empleo del filtro de Hamilton dado en (3).

A pesar de la limitante anteriormente citada, se realizó la prueba de expansión de Huberman y Kandel (1987) para demostrar la hipótesis nula dada por $H_{0}: \alpha=0, \beta=1$. Los resultados se exponen en la tabla 2 .

Gráfica 3. Desviación estándar de 30 días de la variación porcentual de los dos índices estudiados.

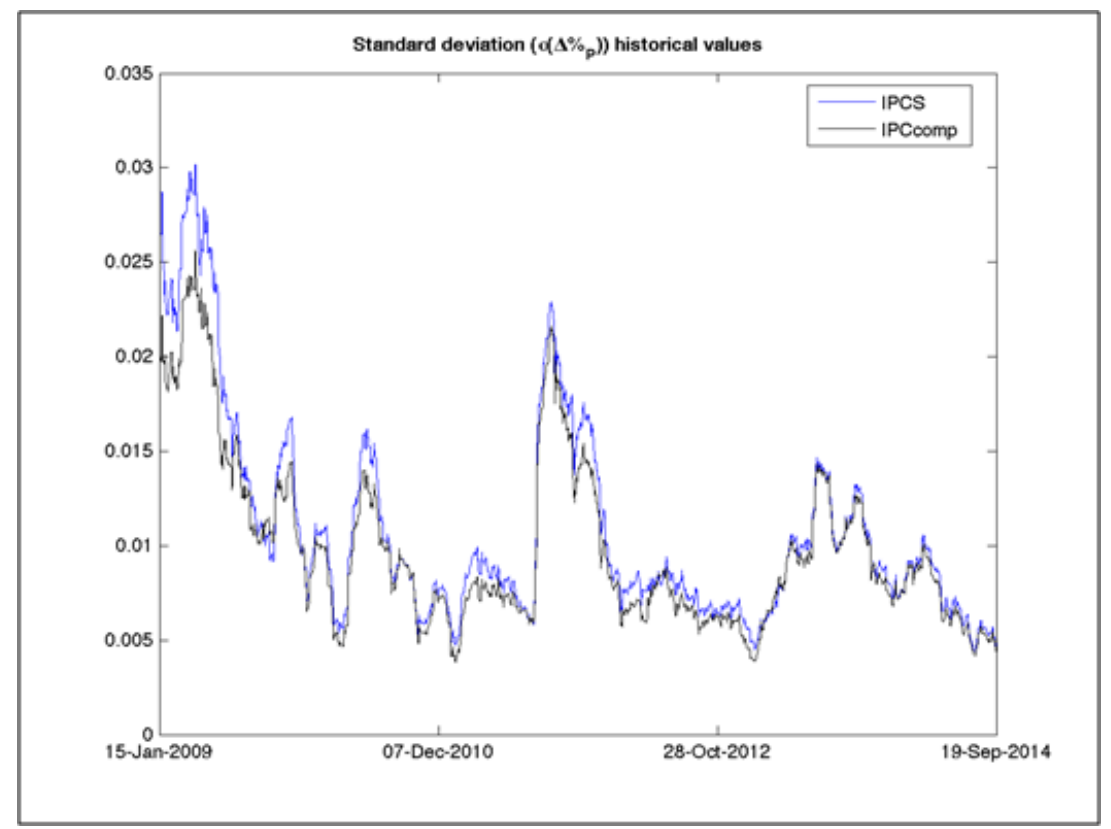

Fuente: Elaboración propia en base a información de las corridas y simulaciones realizadas.

En la misma se observa que la hipótesis nula se sostiene por lo que el desempeño del IPCS y el IPCcomp son prácticamente el mismo. Esto se confirma estadísticamente con el hecho de que el valor de $\beta$ es prácticamente de 1 en términos de magnitud nominal, situación que lleva a 
observar que si se invierte en un portafolio con menor cardinalidad y formado de empresas sustentables, se pueden reducir costos de administración (menos acciones a administrar), llegando a resultados estadísticamente iguales que la inversión convencional. Esto último sin enfrentar una pérdida de eficiencia media-varianza.

Tabla 2. Prueba de expansión de Huberman y Kandel (1987) con la variación porcentual del IPCS como regresada.

\begin{tabular}{|l|l|l|l|}
\hline Coeficiente & Valor & Estadistico $t$ & Probabilidad \\
\hline$\alpha$ & 0.0001 & 1.1887 & $19.6762 \%$ \\
\hline$\beta$ & 1.0951 & 6.4754 & $0.0000 \%$ \\
\hline Estat.F & $22,329.3284$ & Prob. F & $0.0000 \%$ \\
\hline $\mathrm{R}^{2}$ ajust. & 0.9387 & Error std. resid. & $0.3072 \%$ \\
& & &
\end{tabular}

Fuente: Elaboración propia en base a información de las corridas y simulaciones realizadas.

Gráfica 4. Desviación estándar de 30 días de la variación porcentual de los dos índices estudiados.

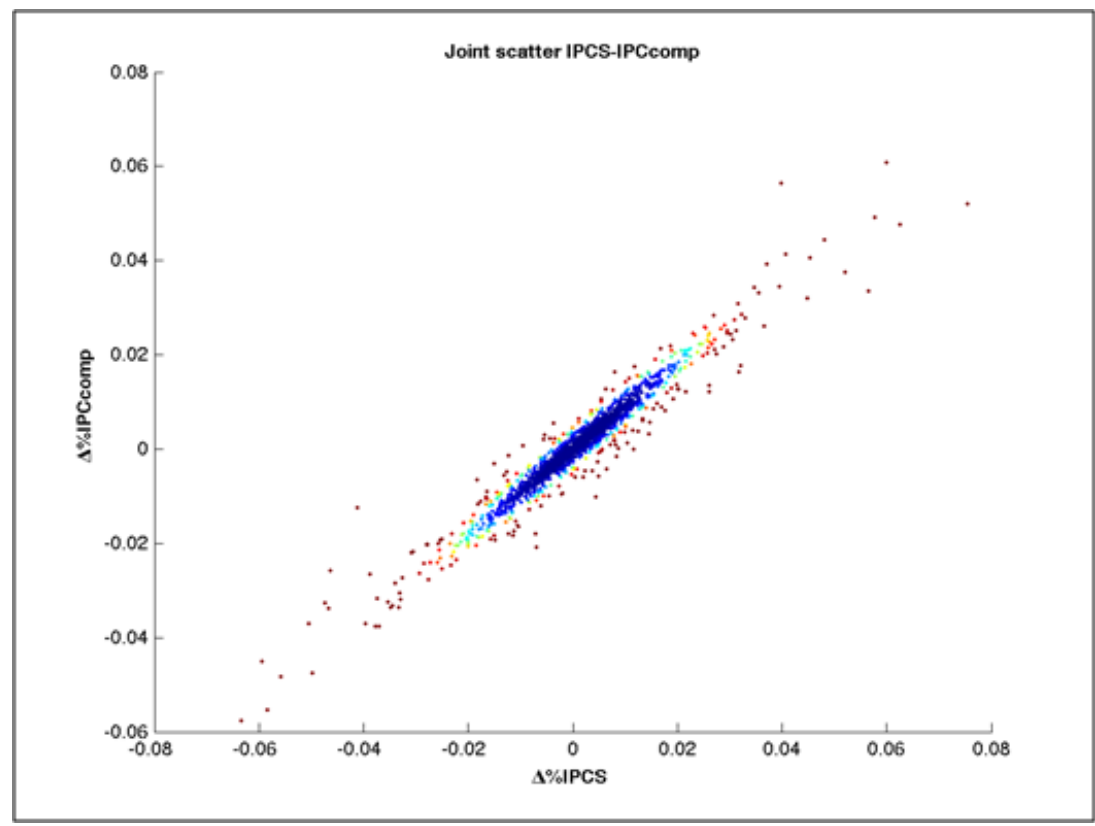

Fuente: Elaboración propia en base a información de la Bolsa Mexicana de Valores y Economática.

Ante los resultados de la tabla 1 y resaltando que las dos últimas pruebas se realizaron para todo el periodo estudiado, es de interés notar que será prudente 
observar el rendimiento esperado y nivel de riesgo en periodos tanto de baja como de alta volatilidad. Para dar una idea inicial se expone en la gráfica 4 un diagrama de dispersión con las variaciones porcentuales del IPCS y el IPCcomp. En la misma se aprecia cómo algunas fechas están significativamente fuera de periodos convencionales (extremos superior derecho e inferior izquierdo).

Tabla 3. Filtro de Hamilton aplicado a las variaciones porcentuales del IPCS y el IPCcomp.

\begin{tabular}{|l|l|l|}
\hline Panel a: Resultados para el IPCS \\
\hline Parámetro & Baja $\sigma$ & Alta $\sigma$ \\
\hline Media $(\mu)$ & $0.0658 \%$ & $0.1434 \%$ \\
\hline Desviación estándar $(\sigma)$ & $0.8008 \%$ & $2.0101 \%$ \\
\hline Matriz de transición de probabilidades & Baja $\sigma$ & Alta $\sigma$ \\
\hline Baja $\sigma$ & $99.4227 \%$ & $1.9138 \%$ \\
\hline Alta $\sigma$ & $0.5773 \%$ & $98.0862 \%$ \\
\hline Panel b: Resultados para el IPCcomp & & \\
\hline Parámetro & Baja $\sigma$ & Alta $\sigma s$ \\
\hline Media $(\mu)$ & $0.0588 \%$ & $0.0907 \%$ \\
\hline Desviación estándar $(\sigma)$ & $0.7043 \%$ & $1.5895 \%$ \\
\hline Matriz de transición de probabilidades & Baja $\sigma$ & Alta $\sigma$ \\
\hline Baja $\sigma$ & $99.3592 \%$ & $1.4347 \%$ \\
\hline Alta $\sigma$ & $0.6408 \%$ & $98.5653 \%$ \\
\hline Panel c: Duración esperada de cada régimen & & \\
\hline Índice & Baja $\sigma s$ & Alta $\sigma$ \\
\hline IPCS & 173.2308315 & 52.25187006 \\
\hline IPCcomp & 156.0555723 & 69.69912039 \\
\hline
\end{tabular}

Fuente: Elaboración propia en base a información de las corridas y simulaciones realizadas.

Dado esto, se corrió el filtro de Hamilton dado en (3) y se tuvieron los resultados observados en la tabla 3 . En el panel a de la misma se aprecian los valores de la media o valor esperado del rendimiento o variación porcentual diaria del IPCS y lo propio se observa en el panel b para el IPCcomp. En términos de riesgo o volatilidad, se confirma lo observado en la gráfica 3. Esto es, que el 
nivel de exposición al riesgo es superior en el IPCS tanto en tiempos normales como en tiempos de crisis. Sin embargo, el rendimiento medio o variación porcentual media es notablemente superior en los dos regímenes. En específico resalta una variación positiva media de $0.14 \%$ en el IPCS contra un de $0.09 \%$ del IPCcomp. Esto sugiere que, en tiempos de crisis, es más rentable realizar inversión sustentable (dada la mayor estabilidad observada en el IPCS respecto al IPCcomp en la gráfica 2).

De manera complementaria, se puede apreciar en el panel c de la tabla 3 que el número de días esperados en que el periodo de crisis duraría es notablemente inferior ( 52 días para el IPCS v.s. 69 para el IPCcomp). Esto se confirma en la gráfica 5, la cual muestra el histórico de las probabilidades filtradas para el régimen de alta volatilidad, así como la media y desviación estándar condicionales tanto para el IPCS como para el IPCcomp.

Gráfica 5 Histórico de probabilidades filtradas, media condicional y desviación estándar condicional al aplicar el Filtro de Halmiton en el IPCS y el IPCcomp.

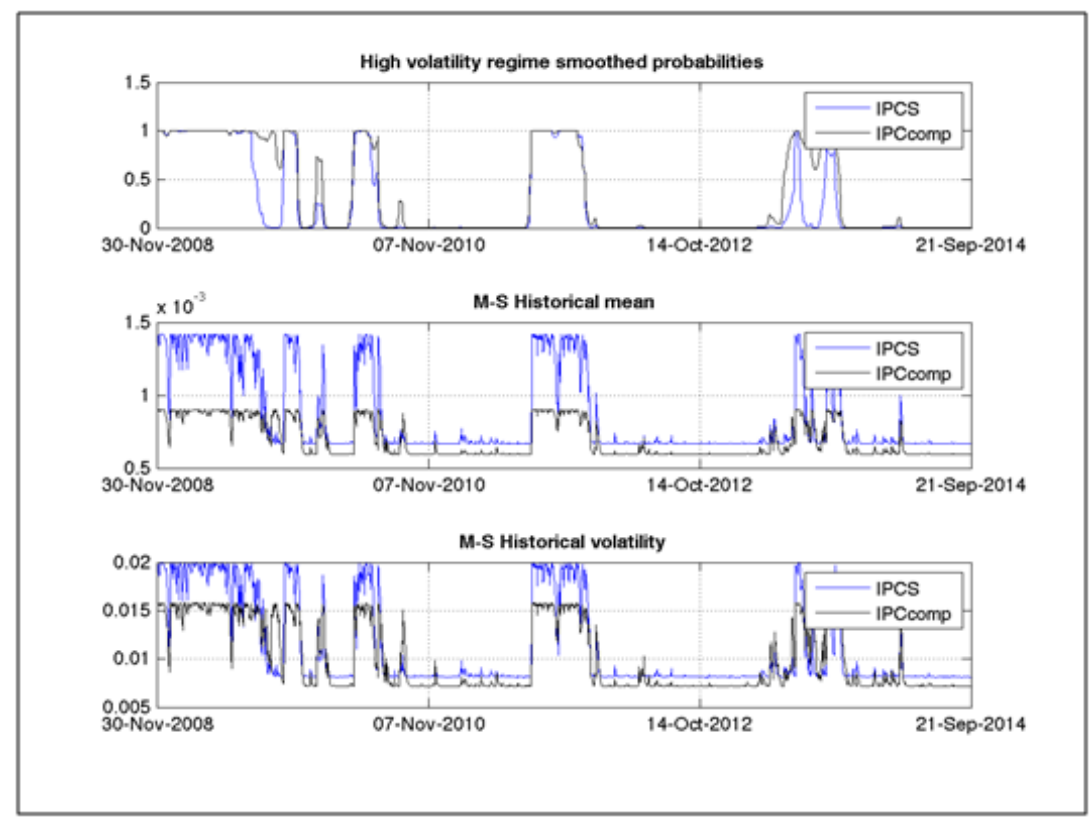

Fuente: Elaboración propia en base a información de las corridas y simulaciones realizadas.

En el panel superior de la gráfica se aprecia que las probabilidades de estar en el régimen de alta volatilidad para el IPCS son bajas en la mayoría de los días observados; siendo lo contrario para el IPCcomp. Este resultado sugiere que la exposición a periodos de crisis es mayor en la inversión convencional que en la sustentable. Esto a pesar de lo observado en la gráfica 3.

Los paneles segundo y tercero de la misma gráfica refrendan lo concluido en las pruebas anteriores: el IPCS tiene niveles sistemáticamente superiores de 
riesgo (dada a la cardinalidad inferior respecto al IPCcomp), acompañado de niveles de desempeño superiores.

Dado esto se aprecia que si bien es cierto que el riesgo observado es mayor en la inversión sustentable en México, las magnitudes observadas son suficientes para no perder eficiencia media-varianza. Esto tanto en escenarios o regímenes de alta como de baja volatilidad, situación que se corrobora con la menor cantidad de días en los que se espera que dure el periodo de crisis en la inversión sustentable.

\section{Conclusiones y consideraciones para futuros trabajos de investigación.}

El presente trabajo es de los primeros en estudiar la inversión sustentable en bolsa para el caso mexicano. Para lograr esto se contrastó al índice IPC sustentable (IPCS) contra el IPC compuesto (IPCcomp). Esto fue así debido a que, en comparación a otras investigaciones citadas en la revisión de bibliografía, existen pocos fondos de inversión sustentable en el país y debido a que la inversión sustentable en bolsa es un tema relativamente nuevo en la República Mexicana.

Ante esta situación y para evitar sesgos en los resultados ante temas como los costos financieros incurridos o la administración propia del fondo, se empleó el comparativo del desempeño de índices.

Dado esto, se utilizaron valores diarios de los índices IPCS e IPCcomp del día 28 de noviembre de 2008 al 20 de septiembre de 2014. Con los mismos se emplearon las variaciones porcentuales para calcular la desviación estándar de 30 días de ambas referencias. Con estos valores se corrió un modelo CAP estándar y una prueba de expansión de Huberman y Kandel (1987). Con estas primeras revisiones, se llegó a la conclusión de que la inversión sustentable en México expone a los inversionistas a niveles de riesgo superiores a los que se tendrían al invertir en una estrategia convencional (como es el replicar un índice de mercado, como el IPCcomp). A pesar de esto, los primeros resultados sugieren que no se pierde desempeño o rendimiento en la inversión socialmente responsable.

Para reforzar lo anterior, se observó en la prueba de expansión que la siguiente hipótesis nula se sostiene: $\alpha=0, \beta=1$. Esto lleva a observar que no solo no se pierde eficiencia media-varianza; sino que también la inversión sustentable (IS) en bolsa y la convencional tienen resultados o comportamientos estadísticamente iguales. Esto lleva a fortalecer los argumentos a favor de la IS debido a que no se perderán rendimientos o desempeño si se decide a favor de un portafolio que cubra las características positivas de ser socialmente responsable, reducir costos financieros y facilitar la operativa de la cartera debido a una menor cantidad de acciones a seguir.

De manera complementaria, se extendió el trabajo a un contexto en donde se separan los datos en periodos de alta y baja volatilidad. Para lograr esto, se aplicó el filtro de Hamilton (1989) para lograr un modelo markoviano para dos regímenes y se confirmó el hecho de que la volatilidad es mayor en el IPCS en ambos regímenes pero el rendimiento esperado es significativamente mayor para el IPCS en escenarios de alta volatilidad. 
Ante esta situación, se tienen las primeras evidencias a favor de la inversión sustentable tanto por desempeño en periodos de alta y baja volatilidad como por beneficios operativos y financieros. Esto sin dejar de lado la relevancia ambiental, social y corporativa que tiene el invertir y apoyar financieramente a empresas calificadas como sustentables.

A pesar de los resultados logrados, es de necesidad mencionar las limitaciones de este avance primigenio en materia de inversión sustentable en México. Como punto de partida se aprecia que la ventana temporal del trabajo es representativa pero es corta en alcance contextual. Es decir, los datos que se tienen son de noviembre de 2008 y periodos de alta volatilidad como el periodo 2007-2008, 2003 y 2001 no son tomados en cuenta, razón por la cual los autores se encuentran en un proceso de recalcular los índices estudiados para periodos de tiempo más largos.

Por otro lado, poco se sabe de los factores que causan que tanto el IPCS como el IPCcomp tengan desempeños estadísticamente iguales. Esto es así por que, a pesar de que se quisieron correr modelos CAPM multifactoriales, no existen en México índices de desempeño (de valor o crecimiento). Ante esto, los autores se encuentran en un proceso de calcular los índices faltantes.

A pesar de lo anterior, estos primeros resultados toman una relevancia para la inversión sustentable por el hecho de que el presente es de los primeros trabajos que estudian el desempeño y beneficios de la misma para México.

De rendir frutos mayores a futuro, la inversión sustentable podrá ser un estilo de inversión bastante adecuado para inversionistas institucionales como fondos de inversión o de pensiones e incluso puede ser una medida de inversión y generación de riqueza, a través de la asignación de capital de inversión a empresas con estándares ecológicos, corporativos y sociales altos. Esto último en beneficio no solo ya de los accionistas; sino de las partes interesadas de la empresa (stakeholders) como son proveedores, empleados, medio ambiente, sociedad y los propios accionistas.

\section{References}

Areal, N., Cortez, M. C., \& Silva, F. (2013). The Conditional Performance of US Mutual Funds over Different Market Regimes: Do Different Types of Ethical Screens Matter?. Financial Markets and Portfolio Management, 27(4), pp. 397-429. doi:10.1007/s11408013-0218-5

Bauer, R., K. Koedijk \& R. Otten (2005). International Evidence on Ethical Mutual fund Performance and Investment Style. Journal of Banking \& Finance, 29(7), pp. 17511767.

Boutin-Dufresne, F., \& P. Savaria (2004). Corporate Social Responsibility and Financial Risk. The Journal of Portfolio Management, 13(1), pp. 57-66.

Capelle-Blancard, G., \& N. Couderc (2009). The Impact of Socially Responsible Investing: Evidence from Stock Index Redefinitions. The Journal of Investing, 18(2), pp. 76-86.

Carhart, M. M. (1997). On Persistence in Mutual Fund Performance. The Journal of Finance, LII(1), pp. 57-82.

Fama, E. F., \& K. R. French (1992). Common Risk Factors in the Returns on Stocks and Bonds. Journal of Financial Economics, 33(1), pp. 3-56.

Freeman, E. (1984). Strategic Management: A Stakeholder Approach. New York: Cambridge university press.

Hamilton, J. D. (1989). A New Approach to the Economic Analysis of Nonstationary Time Series and the Business Cycle. Econometrica, 57(2), pp. 357-384. 
130 Nueva Época REMEF (The Mexican Journal of Economics and Finance)

Huberman, G., \& S. Kandel (1987). Mean-Variance Spanning. The Journal of Finance, 42(4), pp. 873-888. doi:10.2307/2328296

Jansson, M., A. Biel, M. Andersson \& T.Gärling (2011). Investment Style and Perceived Drivers of Adoption of Socially Swedish Institutional Investors. Journal of Investing, 20(3), pp. 118-124.

Langbein, J., \& R. Posner (1980). Social Investing and the Law of Trusts. Michigan Law Review, 79(1), pp. 72-112.

Lee, D. D., \& R. W. Faff (2009). Corporate Sustainability Performance and Idiosyncratic Risk: A Global Perspective. Financial Review, 44(2), pp. 213-237.

Modigliani, F., \& L. Modigliani (1997). Risk-Adjusted Performance. The Journal of Portfolio Management, 23(2), pp. 45-54.

Moskowitz, M. (1972). Choosing Socially Responsible Stocks. Business and Society Review, $1(1)$, pp. $71-75$

Newey, W. K., \& K. D. West (1987). A Simple, Positive Semi-Definite, Heteroskedasticity and Autocorrelation Consistent Covariance Matrix. Econometrica, 55(3), pp. 703-708.

Perlin, M. (2012). About the MS_Regress_Package.pdf. MS_Regress - A Package for Markov Regime Switching Models in Matlab. Retrieved from https://sites.google.com/site/marceloperlin/matlab-code/ms/r_regress-a-package-for-markov-regime-switchingin-matlab

Scholtens, B. (2005). Style and Performance of Dutch Socially Responsible Investment Funds. The Journal of Investing, 14(1), pp. 63-72.

Schr'oder, M. (2004). The Performance of Socially Responsible Investments: Investment Funds and Indices. Financial Markets and Portfolio Management, 18(2), pp. 122-142.

Schr'oder, M. (2007). Is there a Difference? The Performance Characteristics of SRI Equity Indices. Journal of Business Finance \& Accounting, 34(1-2), pp. 331-348.

Statman, M. (2000). Socially Responsible Mutual Funds. Financial Analysts Journal, 56(3), pp. 30-39. 